\title{
Conveying Real-time Ambivalent Feelings through Asymmetric Facial Expressions
}

\author{
Junghyun Ahn ${ }^{1}$, Stéphane Gobron ${ }^{2}$, Daniel Thalmann ${ }^{3}$, and Ronan Boulic ${ }^{1}$ \\ 1 Immersive Interaction Group (IIG), EPFL, Switzerland \\ ${ }^{2}$ Institute of Information and Communication Systems (ISIC), HE-Arc, Switzerland \\ ${ }^{3}$ Institute of Media Innovation (IMI), NTU, Singapore
}

\begin{abstract}
Achieving effective facial emotional expressivity within a realtime rendering constraint requests to leverage on all possible inspiration sources and especially from the observations of real individuals. One of them is the frequent asymmetry of facial expressions of emotions, which allows to express complex emotional feelings such as suspicion, smirk, and hidden emotion due to social conventions. To achieve such a higher degree of facial expression, we propose a new model for mapping emotions onto a small set of 1D Facial Part Actions (FPA)s that act on antagonist muscle groups or on individual head orientation degree of freedoms. The proposed linear model can automatically drive a large number of autonomous virtual humans or support the interactive design of complex facial expressions over time.
\end{abstract}

Keywords: VAD emotional model, facial parameters, asymmetric facial expression, real-time application

\section{Introduction}

This paper proposes a new model for mapping the expression of complex emotions onto a small set of 1D Facial Part Actions (FPA)s acting on antagonist muscle groups or on individual head orientation degree of freedoms. Targeted applications are those requiring reactive on the fly emotional feedback from autonomous characters, for individual interaction as well as for large scale inhabited and shared virtual environments. Therefore, our key motivation is to offer a real-time technique for synthesizing far beyond the six universal emotions [17] and the emotion corpus spanned by the Valence Arousal Dominance (VAD) emotion space $[3,33]$. Indeed, we aim at also rendering those complex emotions resulting in very demonstrative expressions, often with facial asymmetry [35].

We distinguish two types of asymmetry in the expression of emotions. The first one is a systematic asymmetry that depends on the nature of basic emotions such as raising one eyebrow to indicate suspicion [13]. The second type of asymmetry results from the simultaneous combination of two basic emotions, one on each side of the face, when an individual is unsuccessfully trying to hide one emotion by another one, e.g. sadness by fainted joy induced by social conventions (this was described as the "false smile" in [20]). The asymmetry we address 
in this paper is a complementary type of display compared to the upper/lower blend of facial emotion occurring in similar social situations as initially identified in [4] and developed in [26]. The Duchenne smile which is characterized as lacking the eye wrinkling is one illustration of the upper-lower blend of emotions [23, 13]. This latter modality is out of the scope of the present paper.

The next Section recalls the scientific underpinning of facial asymmetry, the prior contributions in the field of real-time facial expression, and the definition of the VAD emotion space. Section 3 then presents the FPAs and the linear mapping from VAD dimension to the corresponding facial deformation. The case of asymmetric expression synthesis is developed in Section 4. Results are displayed in Section 5 before the discussion and conclusion in Section 6 .

\section{Related Works}

\subsection{Asymmetry of human facial expression}

Despite the intrinsic symmetry of the human skeleton and face with respect to the medial/sagittal plane, numerous experimental studies have reported with statistical significance that the expression of emotions were more intense on one side of the face (see the survey from [7]). It was initially reported in [34] that emotion are more intense on the left side, and this, independently of the right or left handedness of the subjects. Given the brain organization of motor control, it characterizes a laterality effect with a greater involvement of the right brain hemisphere for emotional expression. It has been further refined that emotion with negative valence, are more left-biased than positive emotion $[11,5]$. The percentage of left biasing on several emotional terms were also introduced after observing 51 normal adult subjects [6]. There was a different opinion that the left-biased tendency depends on how to settle the experimental environment [36]. However, it has been since further confirmed with experiments with trained judges and quantitative EMG measurements of facial muscles such as zygomatic(cheek to lip corner) or corrugator(near forehead inner brow) $[37,15]$.

Asymmetry also spreads through the different time scales that are at play in the expression of emotions, from the small timing nuance of a smile [20] to the longer lasting emotional coloring that pervades emotional life [13].

\section{$2.2 \quad$ Facial expression synthesis}

A large body of work has been performed on facial expressivity in general $[28,14$, $32,38]$, and in real-time interaction with autonomous virtual humans in particular [40]. Pelachaud and Poggi provide a rich overview of a large set of expressive means to convey an emotional state [31] including head orientation. In [16] facially expressive agents with personality are used for sustained conversations. In this work the facial expression of emotion is obtained by blending predefined faces expressed in terms of MPEG4 facial parameters. Albrecht et al. [2] describe a text-to-speech system capable of displaying emotion by radially interpolating 
key emotions within a 2D emotion space (hence the paper title "mixed feelings" although the proposed approach does not simultaneously integrate two distinct emotions). A recent study on emotion expression through gaze [24] stresses the relationship between a three-dimensional emotion model and multiple postural factors including the head and torso inclination and velocity [29]. However, the asymmetry is not acknowledged as a determinant factor in these studies. In [1], predefined asymmetrical facial expressions are exploited in a barycentric interpolation scheme which requires an important a priori design effort.

\subsection{VAD emotion model}

In the mid 1970's, a number of psychologists challenged the issue of defining a dimensional model of emotion. An emotion space spanned by three independent dimensions has been proposed with slightly different terms depending on the authors $[10,27,3]$. The first axis represents the positivity or negativity of an emotion. The second describes the degree of energy of the emotion. Finally, the third axis indicates the feel of power of emotion. In the present paper, we use the terms Valence, Arousal, and Dominance (VAD) for these three axes.

Based on this 3D emotion space and the related works described above, we set the direction of our contributions as follow: 12 antagonist muscle groups are built from the Action Units (AU) identified in [17]. The normalized activity of the muscle groups is expressed as a linear mapping of the 3D emotion parameters VAD. Ambivalent feelings can be built either automatically from the VAD inputs or through a dedicated interface.

\section{Facial Expressions in VAD Space}

In this Section, we introduce how we relate 3D emotional space to facial expressions. Figure 1 shows the entire pipeline of this VAD face generation.

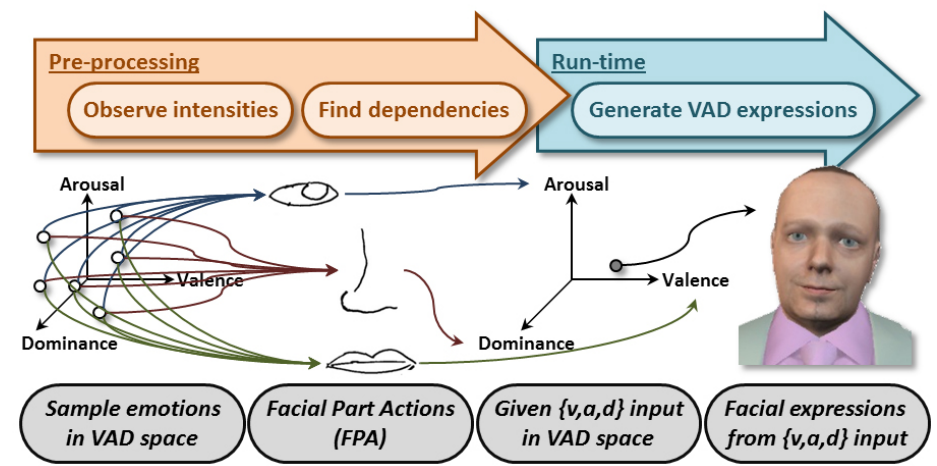

Fig. 1. The overview of facial expression generation from a VAD input. 


\subsection{Sample emotions}

Eighteen sample emotions in VAD space have been selected from the Affective Norms for English Words (ANEW)'s "all subjects table" [9]. In order to take advantage of a substantial amount of previous researches [17, 18, 22, 21], we first selected six basic emotions such as anger(adjective: angry), disgust(disgusted), fear(afraid), happiness(happy), sadness(sad), and surprise(surprised). Twelve other emotional adjectives - anxious, bored, consoled, gloomy, hopeful, indifferent, inspired, overwhelmed, peaceful, pleasant, relaxed, shy - have been also selected for uniform VAD distribution along the emotional space. These VAD coordinates have been taken from [9] and later treated as a basic information for analyzing dependency of each Facial Part Action (FPA) onto emotional axes.

\subsection{Facial Part Actions (FPA)}

According to [8], areas of face that represent emotion are divided into three main components: upper face, lower face, and head orientation. These facial parts are further decomposed into Facial Action Coding System (FACS)'s Action Unit (AU)s [19]. As illustrated in Figure 2, AUs are grouped into twelve Facial Part Actions (FPA)s. The detail of FPAs' moving area is also described in Figure 2.

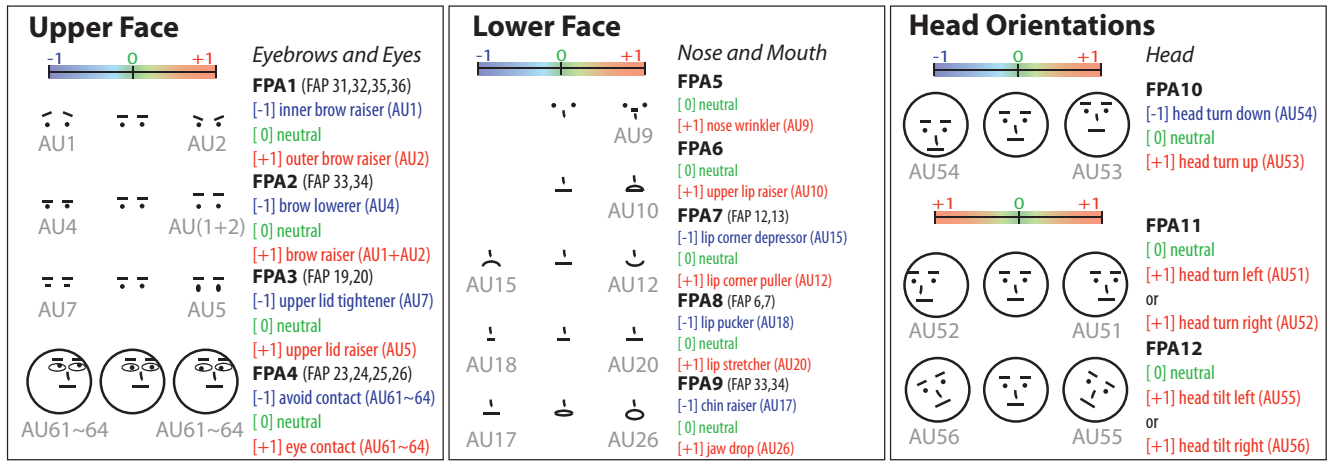

Fig. 2. Illustration of the FPAs' moving range defined by intensities. Except some special cases (FPA5, 6, 11, 12, range $[0,+1]$ ), the intensity range of FPAs are $[-1,+1]$. We also compared FPAs to Facial Animation Parameters (FAP)s introduced in [25].

\section{3 $\quad$ Finding linear dependencies}

We propose to control facial expression by a column vector $\mathbf{y}$ consisting of twelve FPA intensities. The $i^{t h}$ element of $\mathbf{y}$ represents an FPA $i$ intensity driven by a linear function (see Equation 1). A linear model is chosen instead of non-linear model for the following reasons: (1) consistent tendency of intensity along each 
VAD axis; (2) plausible change of emotional expression in between two VADs; and (3) simplified formulation for games. At run time, the input VAD is assigned to $x_{v}, x_{a}$, and $x_{d}$ and generate a vector $\mathbf{y}$ for updating the facial expression.

$$
y_{i}=\beta_{v i} x_{v}+\beta_{a i} x_{a}+\beta_{d i} x_{d}
$$

The coefficients $\beta_{v i}, \beta_{a i}$, and $\beta_{d i}$ are estimated by: (1) gathering FPA intensities (total 12 FPAs $\times 18$ sample emotions) in Table 1; and (2) minimizing residual $\sum_{j=1}^{n}\left\|e_{i j}\right\|^{2}$ (see Equation 2) of multiple linear regression, where $n$ is the number of sample emotions, $y_{i j}$ stands for an observed intensity of FPA $i$ on $j^{\text {th }}$ sample emotion, and $x_{v j}, x_{a j}$, and $x_{d j}$ represent the VAD values of the $j^{\text {th }}$ sample emotion. The regression intercept $\hat{\beta}_{0 i}$ is assigned to 0 , since we wanted to force neutral FPA to zero intensities.

$$
e_{i j}=y_{i j}-\hat{\beta}_{0 i}-\hat{\beta}_{v i} x_{v j}-\hat{\beta}_{a i} x_{a j}-\hat{\beta}_{d i} x_{d j}
$$

The final $\beta$ coefficients are the normalized $\hat{\beta}$ as in Equation 3. From these coefficients, we can generate the intensity vector $\mathbf{y}$ from any VAD input.

$$
\left(\beta_{v i}, \beta_{a i}, \beta_{d i}\right)=\frac{\left(\hat{\beta}_{v i}, \hat{\beta}_{a i}, \hat{\beta}_{d i}\right)}{\left\|\hat{\beta}_{v i}\right\|+\left\|\hat{\beta}_{a i}\right\|+\left\|\hat{\beta}_{d i}\right\|}
$$

\begin{tabular}{|c|c|c|c|c|c|c|c|c|c|c|c|c|c|c|c|}
\hline Emotions & $\mathrm{V}$ & A & D & FPA1 & FPA2 & FPA3 & FPA4 & FPA5 & FPA6 & FPA 7 & FPA8 & FPA9 & FPA10 & FPA11 & FPA12 \\
\hline Afraid & -0.75 & 0.42 & -0.26 & \begin{tabular}{|l|l|}
5 & -0.5 \\
\end{tabular} & 1.0 & 1.0 & -0.5 & 0.5 & 0.0 & -0.5 & 0.0 & 1.0 & 1.0 & 1.0 & 0.0 \\
\hline Angry & -0.54 & 0.54 & 0.14 & 1.0 & -1.0 & 0.5 & 1.0 & 1.0 & 1.0 & -1.0 & 0.0 & -0.5 & 1.0 & 0.0 & 0.0 \\
\hline Disgusted & -0.64 & 0.11 & -0.17 & 1.0 & -1.0 & -1.0 & -0.5 & 1.0 & 1.0 & -1.0 & 0.5 & 0.5 & 1.0 & 1.0 & 0.0 \\
\hline Нарру & 0.80 & 0.37 & 0.41 & -0.5 & 0.0 & 0.0 & 0.5 & 0.0 & 0.5 & 1.0 & 1.0 & 0.0 & -0.5 & 0.0 & 1.0 \\
\hline Sad & -0.85 & -0.22 & -0.39 & -0.5 & 0.0 & -0.5 & -0.5 & 0.0 & 0.0 & -0.5 & -0.5 & -0.5 & -1.0 & 0.0 & 0.0 \\
\hline Surprised & 0.62 & 0.62 & 0.28 & -0.5 & 1.0 & 1.0 & 0.5 & 0.5 & 0.5 & 0.0 & 0.0 & 0.5 & 1.0 & 0.0 & 0.0 \\
\hline Anxious & -0.05 & 0.48 & 0.08 & 0.5 & -0.5 & 0.0 & -0.5 & 0.0 & 0.5 & -0.5 & 0.0 & 0.5 & 0.0 & 0.5 & 0.0 \\
\hline Bored & -0.51 & -0.54 & -0.22 & -0.5 & 0.0 & -0.5 & -0.5 & 0.0 & 0.0 & -0.5 & 0.0 & 0.5 & -0.5 & 0.5 & 0.5 \\
\hline oled & 0.20 & -0.12 & -0.14 & -0.5 & 0.5 & 0.0 & 0.0 & 0.0 & 0.0 & 0. & 0.5 & 0. & -0. & 0.0 & \\
\hline Gloomy & -0.78 & -0.29 & -0.36 & -0.5 & 0.0 & -0.5 & -0.5 & 0.0 & 0.0 & -0.5 & 0.0 & 0.5 & -1.0 & 0.5 & 0.0 \\
\hline Hopeful & 0.53 & 0.20 & 0.10 & -0.5 & 0.5 & 0.5 & 0.5 & 0.0 & 0.0 & 0.5 & 0.5 & 0.0 & 0.5 & 0.0 & 0.5 \\
\hline ent & -0.10 & -0.46 & -0.04 & -0.5 & 0.0 & -0.5 & -1.0 & 0.0 & 0.0 & 0.0 & 0.5 & 0.0 & & & 0.5 \\
\hline Insp & 0.54 & 0.26 & 0.42 & 0.0 & 0.5 & 0.5 & 1.0 & 0.5 & 1.0 & 0.0 & 0.5 & 0.5 & 0.5 & 0.0 & 0.0 \\
\hline Overwhelmed & -0.20 & 0.50 & -0.28 & 0.5 & -0.5 & 0.0 & -0.5 & 0.5 & 0.5 & 0.0 & -0.5 & 0.5 & -0.5 & 0.5 & 0.5 \\
\hline Peaceful & 0.68 & -0.51 & 0.11 & -0.5 & 0.0 & -0.5 & 0.0 & 0.0 & 0.0 & 0.5 & 0.5 & 0.0 & -0.5 & 0.0 & 1.0 \\
\hline Pleasa & 0.82 & 0.19 & & -1.0 & 1.0 & 0.5 & 0.5 & 0.0 & 1.0 & 1. & 1.0 & 0.5 & & 0.0 & 0.5 \\
\hline Relaxed & 0.50 & -0.65 & 0.14 & -1.0 & 0.5 & -0.5 & 0.0 & 0.0 & 0.0 & 0.5 & 0.5 & 0.0 & -0.5 & 0.0 & 0.5 \\
\hline Shy & -0.09 & -0.31 & -0.39 & 0.0 & 0.0 & -0.5 & -1.0 & 0.5 & 0.0 & 0.0 & -0.5 & 1.0 & -0.5 & 0.5 & 0.0 \\
\hline$\beta_{v i}$ & & & & -0.42 & 0.63 & 0.19 & 0.29 & -0.32 & 0.13 & 0.55 & 0.48 & -0.07 & 0.07 & -0.46 & 0.52 \\
\hline$\beta_{a i}$ & & & & 0.46 & -0.02 & 0.49 & 0.25 & 0.59 & 0.51 & -0.08 & -0.00 & 0.35 & 0.56 & 0.06 & -0.22 \\
\hline$\beta_{d i}$ & & & & -0.12 & 0.35 & 0.32 & 0.46 & -0.09 & 0.36 & 0.37 & 0.52 & -0.58 & 0.37 & -0.48 & 0.26 \\
\hline
\end{tabular}

Table 1. An example of FPA intensities for each sample emotion. The VADs of eighteen sample emotions are described from the $2^{\text {nd }}$ to $4^{\text {th }}$ columns. The upper six rows are the six basic emotions; the mid twelve are additional emotions for ensuring a uniform distribution in the VAD space; and the lower three are the $\beta$ coefficients.

As illustrated in Figure 3-Left, Equation 1 describes a plane in a VAD space and separates the area to a positive (plane norm direction) and negative intensity fields. The plane includes the origin $\{0,0,0\}$ of VAD space, which leads to zerointensity on neutral state. 


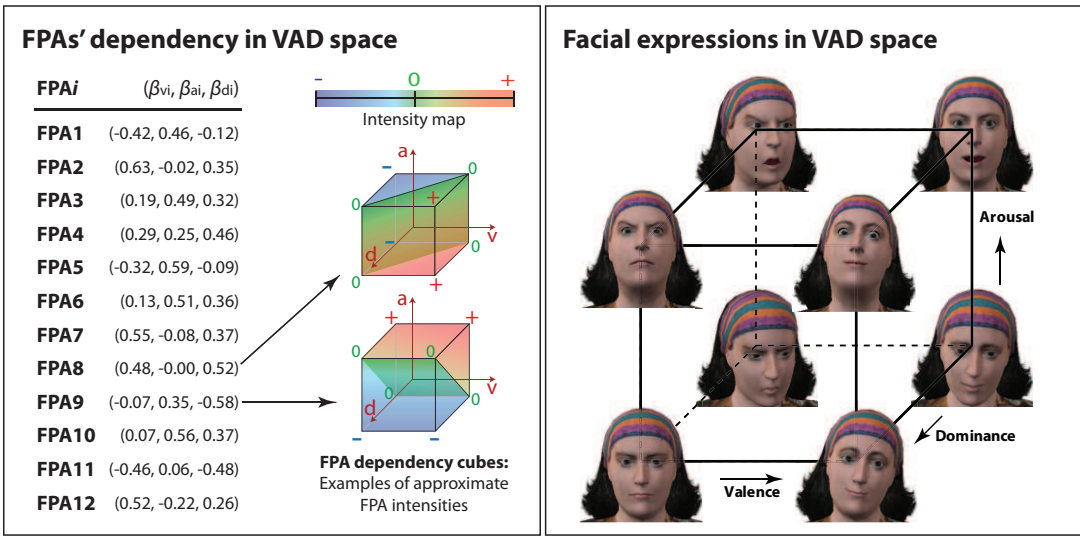

Fig. 3. Left: The FPAs' $\beta$ coefficients (Equation 3) and two examples of FPA dependencies in VAD space. A dependency cube shows how it generates the intensity of corresponding FPA from the VAD space. Green plane (0-corners) is a zero intensity plane as in Equation 1. Red (+) and blue (-) shaded area represents positive and negative intensity field. Right: various facial expressions in a VAD space.

The emotional expression in VAD space can be customized by varying the intensities from Table 1. In this paper, we have exploited the following itemized psychological researches for finding FPA intensities of the six basic emotions. For the additional sample emotions, we manually created facial expressions corresponding to each sample emotion by adjusting FPA values. However, a user study is still needed for the evaluation and validation of these FPA intensities. Eight facial expressions in the corners of the VAD space are demonstrated in Figure 3-Right.

$\diamond$ Six basic emotions and their relation to face are presented in [17].

$\diamond$ Movement of brows can affect emotions such as surprise, fear, sadness, or anger, however there is no affect to disgust or happiness emotion [18].

$\diamond$ Emotions effects on eye lids, lip, and jaw have been observed [22].

$\diamond$ A specific facial part AU 12 (lip movement) has been observed in [21].

\section{$4 \quad$ Facial Asymmetry for Ambivalent Feelings}

There were a number of debates so far on the effect of emotional expression to left/right facial asymmetry, and it still is undetermined. However, if we take a look at the emotional face from pictures, movies or real world, there is no doubt that neither a face, nor its expression are symmetric.

Inspired by those theories (including the previous works described in Subsection 2.1), this paper presents an intuitive interface that facilitates transmission of complex emotional feelings to a virtual character. Among the twelve 
FPAs, five of them are used for asymmetric expressions. We have chosen asymmetric FPAs related to corrugator, orbicularis, and zygomatic muscles, which are mostly observed for left/right asymmetric facial expression from EMG experiments.

Corrugator: AU 1,2, AU 4 (FPA1 and FPA2);

Orbicularis: AU 5,7 (FPA3);

$\diamond$ Zygomatic: AU 12,15, AU 18,20 (FPA7 and FPA8).

\section{$4.1 \quad$ Overall pipeline}

Based on the psychological finding from [5], we designed a pair of inputs which manage independently the left and right (controlled emotion) side of the face. Figure 4 shows the entire pipeline for this asymmetric facial animation. The proposed framework offers two different types of run-time input edited at preprocessing stage: (1) a pair of VAD values with face-edit parameters (see the next sub-section) are pre-defined in face edit phase; or (2) a pair of VAD flows manually designed by an animator. For an efficient face edit process, the proposed interface provides a list of 117 emotional keywords, each linked to VAD value. These keywords were chosen among 1,035 words provided in ANEW table [9].

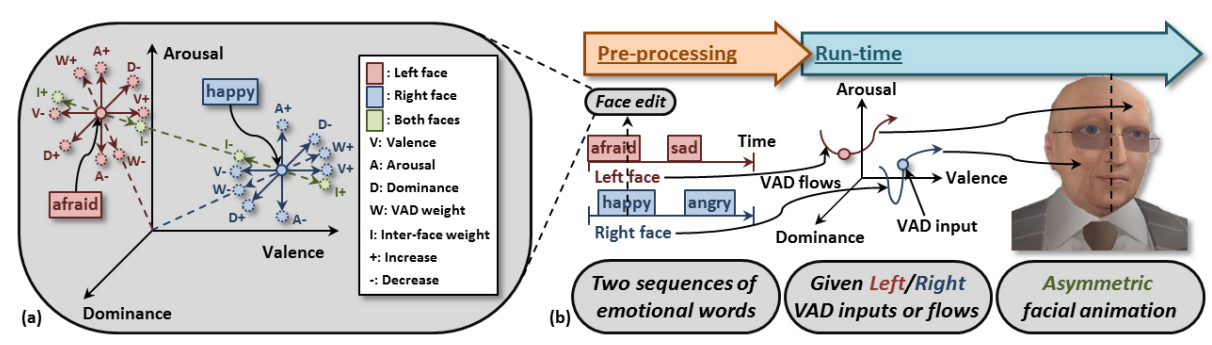

Fig. 4. (a) Asymmetric face-edit parameters; (b) Asymmetric facial animation pipeline.

The right side of the face which expresses a controlled emotion (social conventions), is generated by five right face FPA intensities $y_{1}^{r}, y_{2}^{r}, y_{3}^{r}, y_{7}^{r}$, and $y_{8}^{r}$. An intensity value $y_{i}^{r}$ represents the right face intensity of FPA $i$ (left face intensity is $y_{i}^{l}$ ). In case of symmetric expression, a unique intensity $y_{i}$ is used for both sides of face. Detailed face edit process are explained in the next sub-section.

For the pre-processed VAD flows, we can take advantage of the VAD space, since an emotional keyword can easily be converted to a $3 \mathrm{D}$ vector form (VAD coordinate). It facilitates to interpret given keywords as control points for linear or non-linear VAD interpolation. In both left and right time-lines, an animator can define starting time and duration of an emotional keyword. Those time-line can be played back at run time at the moment when the user gives input. 


\subsection{Asymmetric face-edit parameters}

The first step when building an ambivalent feeling is to choose an emotional keyword for each side of the face. However, we still need to adjust their combination to produce plausible facial expresssions. As illustrated in Figure 4-(a), we defined five asymmetric face-edit parameters for increasing expressivity of the asymmetric face.

From a given asymmetric expression generated simply by two emotional words, the edit parameters focus on the alteration of the current emotional status. Instead of moving a complex mesh structure or rigged face area, the proposed tool provides parameters $(V \pm, A \pm, D \pm$ ) which allow to adjust respectively valence, arousal, and dominance. A VAD weighting factor $(W \pm)$ gives more intense or weak feeling to a given emotional keyword. Moreover, an inter-face weighting factor $(I \pm)$ interpolates or extrapolates feelings between two different keywords.

We also defined a special parameter $b$ (see Equation 4), i.e. a left-bias factor ranged $[-1,+1]$ which increases the left/right intensity of the face. If $b$ is greater than 0 , the expression is left biased. As was mentioned in [36], the facial expression on the left face is more intense when the emotion is negative (valence). Therefore, we applied a weighting factor on the negative emotion [12] by giving extra intensity when the valence is lower than zero. Here the constant $c_{i}$ determines an additional weight of negative valence. Based on our experience 0.25 gives the most plausible results.

$$
\begin{aligned}
y_{i}^{l} & =y_{i}\left(1+b_{i}\left(1-c_{i} x_{v i}\right)\right) \\
y_{i}^{r} & =y_{i}\left(1-b_{i}\left(1-c_{i} x_{v i}\right)\right)
\end{aligned}
$$

\subsection{Ambivalent feelings}

Four examples of ambivalent feelings are illustrated in Figure 5.

The top-left faces shows the smirk expression which combines neutral and happy emotions. This mixed feeling expresses someone who is trying to smile about something that is not funny. After merging the two emotions the left-bias factor $b$ was decreased by 0.3 , resulting in a more intense smile on the right side of the face.

The bottom-left faces combine increased pleasure and decreased jealous emotions. Those two opposite feelings generates a vicious expression as if someone would enjoy negative feelings.

The top-right faces demonstrate a feeling after a certain amount of time from a positive surprise. The arousal of surprised feeling was decreased by 0.6 and again the weighting surprise feeling are again decreased by 0.1 . After these edit operations, a relief expression was generated.

Finally, on the bottom-right, we see a face hiding its anxious feeling and trying to pretend to be cool. This mixed feeling was combined by anxious and relaxed emotion. All four operations help to intensify relaxed feeling against anxious feeling. 


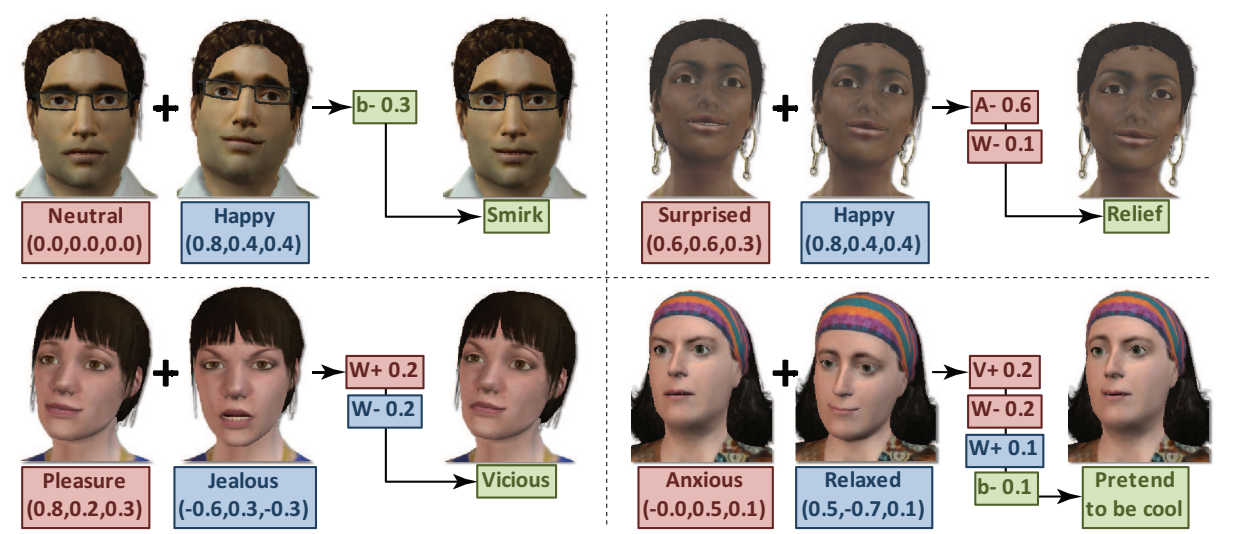

Fig. 5. Four ambivalent feelings generated by the proposed framework. The VAD coordinates are described below the emotional keywords. Red boxes represent emotion and edit parameters applied to the left face; Blue and green boxes correspond to the right face and both faces, respectively; $W+, W-, A+, A-$, and $V+$ are face edit operators illustrated in Figure $4(\mathrm{a}) ; b$ - is the left-bias parameter defined in Equation 4.

\section{$5 \quad$ Applications and Experiment}

As we demonstrates in a video provided in http://youtu.be/ycUoZ4jAU_E our approach can easily be plugged into a pre-existing character animation sequence. As depicted in Figure 6-Left, the role of the elements of the FPA vector $\mathbf{y}$ are used as delta intensities of animated postures. In this way, we are not only able to modify the emotional feeling of the existing animation, but also able to express complex feeling of a virtual character at run time. Moreover, thanks to a simple VAD interface and the emotional keywords, the proposed framework can easily be embedded into a game or VR application with text sentiment analysis [39]. It helps to efficiently simulate autonomous affective characters in an online game.

As we focus on a real-time environment such as games or VR applications, we measured the display rate of a scene with 200 characters. As depicted in Figure 6-Right, the users' emotional VAD input are continuously given at run time. With an NVidia GTX 460 1-GB hardware, we were able to simulate and edit facial expression at $>60 \mathrm{fps}$.

\section{Conclusion and Discussion}

In this paper, we presented a novel approach enlarging the spectrum of facial expressions that allows the synthesis of emotional asymmetric facial expression from the input of a 3D emotional model. In order to achieve this, we examined a number of literature surveys on social and neuro psychology for: (1) choosing a suitable 3D emotional model; (2) identifying a model of the animation of each facial part; and (3) exploiting the effect of asymmetry for emotion expression. 

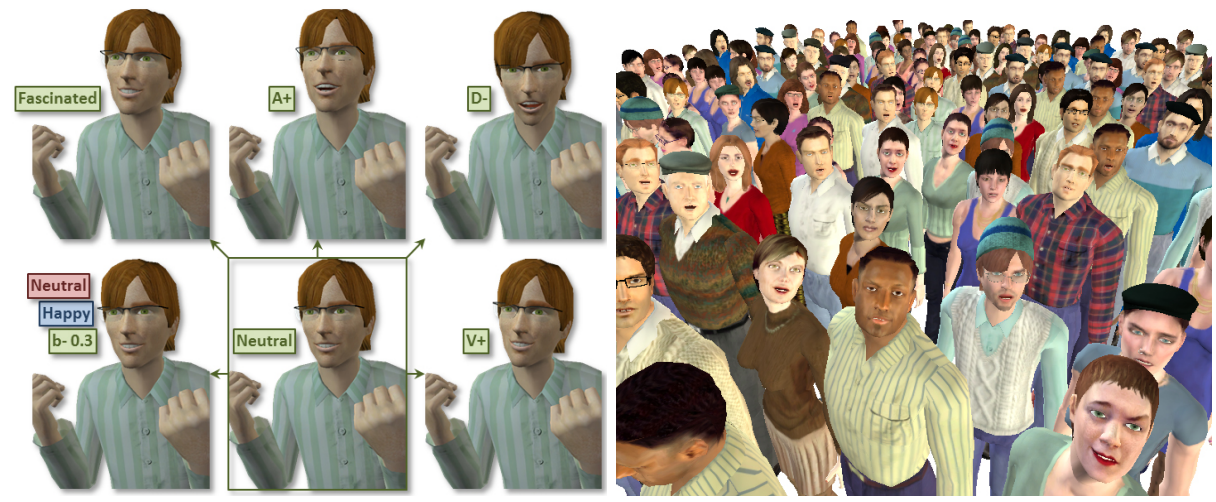

Fig. 6. Left: Emotion variation to a pre-existing "excited" animation. We applied different face edit parameters onto the original ("neutral" in terms of delta intensity) animation; Right: complex facial expression simulated in a crowd scene.

We retained eighteen emotion samples evenly distributed in the VAD model space and grouped the facial AUs into twelve FPAs representing antagonist muscle groups. Each FPA's dependency on emotional dimensions has been analyzed independently by observing the emotion samples. From the analysis, the intensity of each FPA movement has been modeled as a linear function and exploited for facial expression. In some cases an additional linear inequality constraint has been specified. We estimate that the proposed approach will not only assist the game players who want to transfer their emotion into their avatar, but also help the designers of embodied conversational agents to convey complex emotions through the added nuances made possible with the proposed emotion model.

Comparison with earlier references in facial expression $[31,30,24]$ highlights the lack of considering the left/right asymmetry in the emotional expressions for real-time conversational agents. The model we propose induces a very little overhead in terms of computing cost compared to the use of the $3 \mathrm{D}$ emotion model for a real-time application with multiple avatars and autonomous agents.

However, there are still limitations and further improvements to do on this approach. We tried to mix two different emotional representations (categorical and dimensional approach), which could raise issues from psychologists. In terms of facial expression, the proposed method did not consider an appropriate synthesis for the lip movement of a highly aroused (mouse-opened expressions) talking character. Moreover, we took direction to simplify the complex mechanism of emotional expression as a linear model to provide an efficient way of controlling facial expressions. We may explore nonlinear model to better reflect the interactions between simultaneous or successive conflicting emotions.

In the near future, we plan to: (1) exploit more asymmetric facial expression such as "sneer"; (2) conduct more experiments with subjects for evaluation and validation; and (3) increase the number of sample emotions for finer analysis. 


\section{Acknowledgements}

The authors wish to thank Mr. Quentin Silvestre, Mr. Olivier Renault, Ms. Mireille Clavien, Dr. Janusz Holyst, Dr. Arvid Kappas, and Ms. Kamila Kowalska for their collaboration on motion capture, animation, and avatar modeling. This work was supported by a European Union grant by the 7th Framework Programme, Theme 3: Science of complex systems for socially intelligent ICT, which is part of the CYBEREMOTIONS Project (Contract 231323).

\section{References}

1. Ahn, J., Gobron, S., Silvestre, Q., Thalmann, D.: Asymmetrical facial expressions based on an advanced interpretation of two-dimensional russells emotional model. In: ENGAGE summer school workshop (2010)

2. Albrecht, I., Schröder, M., Haber, J., Seidel, H.P.: Mixed feelings: expression of non-basic emotions in a muscle-based talking head. VR 8(4), 201-212 (2005)

3. Averill, J.R.: A semantic atlas of emotional concepts. JSAS Catalog of Selected Documents in Psychology 5(330), 1-64 (1975)

4. Bassili, J.N.: Emotion recognition: The role of facial movement and the relative importance of upper and lower areas of the face. Journal of Personality and Social Psychology 37(11), 2049-2058 (1979)

5. Borod, J., Haywood, C., Koff, E.: Neuropsychological aspects of facial asymmetry during emotional expression: A review of the normal adult literature. Neuropsychology Review 7(1), 41-60 (1997)

6. Borod, J.C., Caron, H.S.: Facedness and emotion related to lateral dominance, sex and expression type. Neuropsychologia 18(2), 237-241 (1980)

7. Borod, J.C., Koff, E., Yecker, S., Santschi, C., Schmidt, J.M.: Facial asymmetry during emotional expression: Gender, valence, and measurement technique. Neuropsychologia 36(11), 1209-1215 (1998)

8. Boucher, J.D., Ekman, P.: Facial areas and emotional information. Journal of Communication 25(2), 21-29 (1975)

9. Bradley, M.M., Lang, P.J.: Affective norms for english words (anew): Stimuli, instruction manual and affective ratings. C-1, University of Florida, Center for Research in Psychophysiology, Gainesville (1999)

10. Bush, L.E.: Individual differences multidimensional scaling of adjectives denoting feelings. Journal of Personality and Social Psychology 25(1), 50-57 (1973)

11. Campbell, R.: The lateralisation of emotion: A critical review. International Journal of Psychology 17(1-4), 211-229 (1982)

12. Chmiel, A., Sienkiewicz, J., Paltoglou, G., Buckley, K., Thelwall, M., Holyst, J.A.: Negative emotions accelerating users activity in bbc forum. CoRR 1011.5459 (2010)

13. Cowie, R.: Perceiving emotion: towards a realistic understanding of the task. Philosophical Transactions of The Royal Society B 364, 3515-3525 (2009)

14. Deng, Z., Neumann, U.: Data-Driven 3D Facial Animation. Springer (2007)

15. Dimberg, U., Petterson, M.: Facial reactions to happy and angry facial expressions: Evidence for right hemisphere dominance. Psychophysiology 37(5), 693-696 (2000)

16. Egges, A., Kshirsagar, S., Magnenat-Thalmann, N.: Generic personality and emotion simulation for conversational agents. CAVW 15, 1-13 (2004)

17. Ekman, P.: Universals and cultural differences in facial expressions of emotions. In: Nebraska Symposium on Motivation. pp. 207-283. Univ. of Nebraska Press (1971) 
18. Ekman, P.: About brows: Emotional and conversational signals. In: von Cranach, M., Foppa, K., Lepenies, W., Ploog, D. (eds.) Human Ethology. pp. 169-248. Cambridge: Cambridge University Press (1979)

19. Ekman, P., Friesen, W.V.: Facial action coding system. CA: Consulting Psychologists Press (1978)

20. Ekman, P., Friesen, W.V.: Felt, false, and miserable smiles. Journal of Nonverbal Behavior 6(4), 238-252 (1982)

21. Ekman, P., Friesen, W.V., Ancoli, S.: Facial signs of emotional experience. Journal of Personality and Social Psychology 39(6), 1125-1134 (1980)

22. Ekman, P., Roper, G., Hager, J.C.: Deliberate facial movement. Child Development 51(3), 886-891 (1980)

23. Krumhuber, E., Kappas, A.: Moving smiles: The role of dynamic components for the perception of the genuineness of smiles. Journal of Nonverbal Behavior 29(1), 3-24 (2005)

24. Lance, B., Marsella, S.: Glances, glares, and glowering: how should a virtual human express emotion through gaze? AAMAS 20(1), 50-69 (2010)

25. Magnenat-Thalmann, N., Thalmann, D.: Handbook of Virtual Humans. Wiley (2004)

26. Martin, J.C., Niewiadomski, R., Devillers, L., Buisine, S., Pelachaud, C.: Multimodal complex emotions: Gesture expressivity and blended facial expressions. International Journal of Humanoid Robotics 3(3), 269-291 (2006)

27. Mehrabian, A., Russell, J.A.: An Approach to Environmental Psychology. The MIT Press (1974)

28. Parke, F.I., Waters, K.: Computer Facial Animation. AK Peters (2008)

29. Paterson, H.M., Pollick, F.E., Sanford, A.J.: The role of velocity in affect discrimination. In: Proceedings of the 23rd Annual Conference of the Cognitve Science Society. pp. 756-761 (2001)

30. Pelachaud, C.: Modelling multimodal expression of emotion in a virtual agent. Philosophical Transactions of The Royal Society B 364(1535), 3539-3548 (2009)

31. Pelachaud, C., Poggi, I.: Subtleties of facial expressions in embodied agents. Journal of Visualization and Computer Animation 13(5), 301-312 (2002)

32. Pighin, F., Hecker, J., Lischinski, D., Szeliski, R., Salesin, D.H.: Synthesizing realistic facial expressions from photographs. In: SIGGRAPH '98. pp. 75-84. ACM, New York, NY, USA (1998)

33. Russell, J.A.: A circumplex model of affect. Journal of Personality and Social Psychology 39(6), 1161-1178 (1980)

34. Sackeim, H.A., Gur, R.C., Saucy, M.C.: Emotions are expressed more intensely on the left side of the face. Science 202(4366), 434-436 (1978)

35. Schatz, H.: In CHARACTER. Bulfinch Press (2006)

36. Schiff, B.B., MacDonald, B.: Facial asymmetries in the spontaneous response to positive and negative emotional arousal. Neuropsychologia 28(8), 777-785 (1990)

37. Schwartz, G.E., Ahern, G.L., Brown, S.L.: Lateralized facial muscle response to positive and negative emotional stimuli. Psychophysiology 16(6), 561-571 (1979)

38. Tena, J.R., De la Torre, F., Matthews, I.: Interactive region-based linear 3d face models. ACM Trans. Graph. 30(4), 76:1-76:10 (Jul 2011)

39. Thelwall, M., Buckley, K., Paltoglou, G., Cai, D., Kappas, A.: Sentiment in short strength detection informal text. JASIST 61(12), 2544-2558 (Dec 2010)

40. Vinayagamoorthy, V., Gillies, M., Steed, A., Tanguy, E., Pan, X., Loscos, C., Slater, M.: Building expression into virtual characters. In: Eurographics Conference State of the Art Report (2006) 